\title{
Proteoglycans in granulomatous lung diseases
}

\author{
E.S. Bensadoun*, A.K. Burke*, J.C. Hogg**, C.R. Roberts*
}

Proteoglycans in granulomatous lung diseases. E.S. Bensadoun, A.K. Burke, J.C. Hogg, C.R. Roberts. OERS Journals Ltd 1997.

ABSTRACT: In nongranulomatous fibrotic lung conditions, we have recently shown that early collagen synthesis by myofibroblasts occurs in an extracellular matrix rich in the proteoglycan versican. We hypothesized that versican is associated with the process of collagen synthesis resulting from chronic inflammation. In this study, we examined the localization of proteoglycans and collagen in the granulomatous lung conditions, sarcoidosis, extrinsic allergic alveolitis (EAA) and tuberculosis (TB).

Tissue from individuals with sarcoidosis $(n=6)$, EAA $(n=4)$ and TB $(n=2)$ was examined for glycosaminoglycans and collagen using histochemistry, and for versican, decorin, biglycan, hyaluronan, type I procollagen and $\alpha$-smooth muscle actin using immunohistochemistry.

The results showed that in sarcoidosis, EAA and TB, the rim of connective tissue surrounding granulomas contained glycosaminoglycans and collagen, and that glycosaminoglycan staining corresponded to localization of versican. Versican-rich zones contained myofibroblasts that stained intracellularly for type I procollagen. Hyaluronan was found diffusely throughout the matrix. Decorin was localized intracellularly in the epithelioid cells of granulomas and some myofibroblasts.

We conclude that the deposition of versican is specific to the early remodelling process in both granulomatous and nongranulomatous lung diseases. In both forms of lung fibrosis, regardless of the nature of the driving inflammatory process, collagen synthesis takes place in a versican-rich provisional matrix. These results suggest that versican may influence the progression of the repair process following many different types of lung injury.

Eur Respir J 1997; 10: 2731-2737.
Depts of *Medicine and **Pathology, University of British Columbia Pulmonary Research Laboratory, University of British Columbia, St Paul's Hospital, Vancouver, Canada.

Correspondence: C.R. Roberts, University of British Columbia Pulmonary Research Laboratory, University of British Columbia, St Paul's Hospital, 1081 Burrard Street, Vancouver, British Columbia, Canada, V6Z $1 \mathrm{Y} 6$

Keywords: Extrinsic allergic alveolitis, proteoglycans, pulmonary fibrosis, sarcoidosis, versican

Received: January 201997

Accepted after revision September 27 1997

This work was supported by grants from the B.C. Lung Association and the M.R.C. of Canada. C.R. Roberts is an M.R.C. of Canada/B.C. Lung Association Research Scholar.
Granulomatous lung inflammation is characterized by the accumulation of mononuclear phagocytes leading to the formation of discrete structures, composed of a central core of epithelioid cells, occasional multinucleated giant cells and a surrounding rim of lymphocytes, macrophages, fibroblasts and connective tissue [1]. These granulomatous lesions are typical of lung diseases such as sarcoidosis, extrinsic allergic alveolitis (EAA), and tuberculosis (TB). In the end stages the cellular elements of the granulomas are gradually replaced by collagen, resulting in a pattern of fibrosis that is indistinguishable from the end stages of nongranulomatous fibrotic lung diseases [2]. While an increase in collagen type I and III occurs as part of the process [3, 4], little is known about changes in other extracellular matrix (ECM) components such as proteoglycans and glycosaminoglycans in granulomatous lung diseases and how they compare with changes in nongranulomatous lung inflammation.

Proteoglycans are a group of glycoproteins that each consist of a core protein covalently linked to a variable number of negatively charged glycosaminoglycan chains. Proteoglycans have a number of biological functions in the ECM, including influencing tissue mechanics, tissue water homeostasis, cell adhesion, cell migration, and the biological activities of several growth factors [5]. We have recently localized proteoglycans and the glycosamino- glycan hyaluronan in the normal adult human lung and in nongranulomatous lung conditions, such as idiopathic pulmonary fibrosis (IPF), the adult respiratory distress syndrome (ARDS) and bronchiolitis obliterans organizing pneumonia (BOOP) [6]. Our data showed that the proteoglycan versican was a consistent and abundant component of the characteristic lesions of each of these conditions. Versican deposition appeared to be an early event in the repair process and early collagen synthesis was localized to myofibroblasts in versican-rich, collagenpoor domains. We hypothesized that versican is associated with the process of collagen synthesis resulting from chronic inflammation. In this study we investigated the association between proteoglycans and collagen synthesis in granulomatous lung diseases.

\section{Materials and methods}

\section{Patients}

The studies were performed on lung tissues entered into the University of British Columbia Pulmonary Research Laboratory tissue registry during the period 1977-1993. 
Cases with a histological diagnosis of sarcoidosis $(n=6)$ and EAA $(n=4)$ were entered into the study after infectious aetiologies were excluded. Lung tissue was also obtained from two patients with confirmed Mycobacterium tuberculosis infection.

Two of the six cases of sarcoidosis were asymptomatic and had normal pulmonary function tests (PFTs) while the remaining four cases had symptoms and PFTs showing either restriction, a reduction in diffusing capacity, or both. Radiographically, four cases represented stage III disease and two cases stage II disease. Clinical data for these patients are summarized in table 1 . The EAA cases had symptoms of 1-6 months duration and the three patients with available PFTs all had restrictive defects. None of the sarcoidosis or EAA patients were taking steroids at the time of their open lung biopsies.

\section{Histology}

All lung tissues were fixed in buffered formalin, embedded in paraffin and serially sectioned at a thickness of $5 \mu \mathrm{m}$. Individual sections were stained with: haematoxylin and eosin (H\&E) to visualize the overall architecture; alcian blue to visualize glycosaminoglycans; and picrosirius red to visualize collagen. All sections for histochemical and immunohistochemical staining were processed and stained concurrently.

\section{Antibodies for immunohistochemistry}

A rabbit polyclonal antibody to human versican was generously provided by E. Ruoslahti and R. Le Baron of the La Jolla Cancer Center [7]. Rabbit polyclonal anti-peptide antibodies to human decorin (LF-30) [8] and biglycan (LF-51) [9] were kindly donated by L.W. Fisher of the National Institutes for Health (NIH) (Bethesda, MD, USA). Cells actively synthesizing type I collagen were localized using a rat monoclonal antibody to the aminoterminal end of human type I procollagen (Chemicon Inc., Temecula, CA, USA). A mouse monoclonal antibody to alpha smooth muscle actin ( $\alpha$-SMA) (1A4) (Sigma Chemical Co., St Louis, MO, USA) was used to detect the presence of myofibroblasts [10]. All antibodies were used at empirically determined optimal dilutions [6] in Tris-buffered saline, $\mathrm{pH} 7.5$ containing $2 \%$ bovine serum albumin (TBS-BSA) (ICN Biochemicals, Cleveland, OH, USA).

\section{Immunohistochemistry}

Sections for immunostaining with versican, decorin and biglycan were dewaxed and then pretreated for $30 \mathrm{~min}$ at $37^{\circ} \mathrm{C}$ with $1 \mathrm{U} \cdot \mathrm{mL}^{-1}$ chondroitinase $\mathrm{ABC}$ (Sigma) in 0.1 $\mathrm{M}$ Tris- $\mathrm{HCl}, 50 \mathrm{mM}$ calcium acetate, $0.01 \%$ BSA, $\mathrm{pH}$ 7.3. This procedure increases staining by all three proteoglycan antibodies [6]. Sections were then blocked with $10 \%$ normal goat serum in TBS-BSA for $1 \mathrm{~h}$ followed by a $2 \mathrm{~h}$ incubation with the primary antibodies at the following dilutions: versican (1/250), decorin (1/200) and biglycan (1/500). Type I procollagen immunostaining required a pretreatment with $1 \%$ trypsin (Sigma) in TBS for $20 \mathrm{~min}$ at room temperature, after which sections were blocked with $10 \%$ normal rat serum in TBS-BSA for $1 \mathrm{~h}$ and incubated for $2 \mathrm{~h}$ with the primary antibody at a dilution of $1 /$ 500 . Sections to be stained for $\alpha$-SMA were blocked with $10 \%$ normal rabbit serum in TBS-BSA for $1 \mathrm{~h}$ followed by a $2 \mathrm{~h}$ incubation with the primary antibody at $1 / 500$. Negative controls were incubated with equivalent concentrations of either normal rat serum or mouse immunoglobulin $(\mathrm{Ig}) \mathrm{G}$ in place of the primary antibodies.

After incubation with the primary antibodies, all sections were washed and incubated for $30 \mathrm{~min}$ in a $1 / 20$ dilution in TBS-BSA of either goat anti-rabbit IgG (Serotec, Kidlington, UK), goat anti-rat IgG (Caltag Laboratories Inc., San Francisco, CA, USA), or rabbit anti-mouse IgG (Dako, Glostrup, Denmark), as appropriate. Sections were then washed and the primary antibody-secondary antibody complexes were localized by incubation for 30 $\min$ in a $1 / 50$ dilution in TBS-BSA of the appropriate alkaline phosphatase-immunoglobulin conjugate (i.e., alkaline phosphatase rabbit IgG conjugate (Serotec), alkaline phosphatase rat IgG conjugate (Serotec) or alkaline phosphatase mouse IgG complex (Dako)). Napthol-ASBI-phosphate/new fuchsin (Sigma) was used as an alkaline phosphatase substrate to visualize the antibody-target complexes as a red precipitate.

\section{Localization of hyaluronan}

Hyaluronan was localized in tissue sections using the hyaluronan-binding protein, link protein, which was purified from bovine nasal cartilage, biotinylated and used according to techniques previously described [6]. Briefly, sections to be stained for hyaluronan were blocked for $2 \mathrm{~h}$ in TBS-BSA and incubated for $4 \mathrm{~h}$ with $200 \mu \mathrm{g} \cdot \mathrm{mL}^{-1}$ biotinylated link protein in TBS-BSA at room temperature.

Table 1. - Clinical data for sarcoidosis patients

\begin{tabular}{|c|c|c|c|c|c|}
\hline $\begin{array}{l}\text { Patient } \\
\text { No. }\end{array}$ & $\begin{array}{l}\text { Age } \\
\text { yrs }\end{array}$ & Sex & $\begin{array}{c}\text { Duration of } \\
\text { respiratory symptoms }\end{array}$ & $\begin{array}{l}\text { Radiographic } \\
\text { stage }\end{array}$ & $\begin{array}{l}\text { Pulmonary function } \\
\text { tests }\end{array}$ \\
\hline 1 & 78 & $\mathrm{M}$ & Asymptomatic & III & Normal \\
\hline 2 & 37 & M & Asymptomatic & III & Normal \\
\hline 3 & 29 & $\mathrm{~F}$ & 12 months & II & Mild restrictive defect \\
\hline 4 & 62 & $\mathrm{~F}$ & 2 months & II & Mild restrictive defect, reduced $T \mathrm{~L}, \mathrm{CO}$ \\
\hline 5 & 52 & M & 2 months & III & Normal TLC, reduced $T \mathrm{~L}, \mathrm{CO}$ \\
\hline 6 & 28 & $\mathrm{~F}$ & 6 months & III & Normal TLC, reduced TL,CO \\
\hline
\end{tabular}

M: male; F: female; TLC: total lung capacity; TL,CO: transfer factor of the lung for carbon monoxide. Radiographic staging system: stage 0: normal chest radiograph; stage I: hilar and/or mediastinal adenopathy without parenchymal involvement; stage II: hilar and/or mediastinal adenopathy with parenchymal involvement; stage III: parenchymal involvement alone. 
These sections were washed and incubated with 1 in 100 streptavidin conjugated to alkaline phosphatase (Pierce, Rockford, IL, USA) in TBS-BSA for $1 \mathrm{~h}$ at room temperature. The alkaline phosphatase was developed using naphthol-AS-BI-phosphate/new fuchsin (Sigma) as a substrate.

After incubation of sections with alkaline phosphatase substrate, all sections stained were counterstained with Gill's haematoxylin, dehydrated in ethanol, mounted in Entellan (BDH Chemicals, Toronto, Ontario, Canada) and examined by light microscopy.

\section{Results}

\section{Sarcoidosis}

The histology revealed single and coalescing non-necrotizing granulomas, adjacent to which the interstitium was minimally thickened by an inflammatory infiltrate (fig. 1A). Alcian blue staining showed abundant glycosaminoglycans in the rim of loose ECM around the granulomas. The central core of epithelioid cells and the adjacent interstitium did not stain for glycosaminoglycans (fig. 1B). Immuno-staining for specific proteoglycans showed that versican was present in the outer rim of ECM but not in the ECM of the central core, in areas of dense collagen staining, or the adjacent interstitium. Versican staining was highly concor-dant with glycosaminoglycan staining (fig. 1C). In contrast, normal human adult lung tissue stains minimally for glycosaminoglycans or versican, using the same techniques [6]. A concentric pattern of collagen deposition was noted within the versican-rich rim around the granulomas (fig. 1B). Hyaluronan was abundant throughout the ECM surrounding granulomas, the ECM of the central core and in the thickened interstitium (fig. 1E). The ECM stained minimally for decorin or biglycan, but the ECM in areas of dense collagen deposition stained more intensely for decorin and biglycan (not shown). Intense intracellular staining for decorin was seen within the epithelioid cells of the central core, and in some myofibroblasts, alveolar macrophages and epithelial cells (fig. 1D). Cells within the versican-rich zone were positive for $\alpha$-SMA (fig. 1F) and type I procollagen (fig. 1G) which identified them as myofibro-blasts synthesizing type I collagen.

\section{Extrinsic allergic alveolitis}

The cases of EAA were characterized by patchy interstitial pneumonitis, minimal fibrosis, and loosely formed granulomas (fig. 2A). Alcian blue staining demonstrated glycosaminoglycans within the thickened interstitium (fig. 2B) and in the rim of ECM surrounding well-formed granulomas (not shown) in a pattern similar to that seen in sarcoidosis. In the thickened interstitium, versican localization was concordant with glycosaminoglycan staining (fig. 2C). Versican-rich areas within the thickened interstitium and around granulomas showed minimal collagen staining (fig. 2B), but cells positive for type I procollagen and $\alpha$-SMA were seen within the versican-rich zones (not shown). Hyaluronan was present throughout the interstitium. The ECM showed minimal staining for decorin and biglycan, although, decorin was found intracellularly within epithelioid cells of granulomas, in myofibroblasts and in some epithelial cells, in a pattern similar to sarcoidosis (not shown).

\section{Tuberculosis}

The histology showed granulomatous inflammation characterized by the presence of caseous necrosis in some of the granulomas (fig. 2D), glycosaminoglycans (fig. 2E) and versican (fig. 2F) co-localized to the rim of ECM surrounding the noncaseating granulomas in a pattern similar to that seen in sarcoidosis. Noncaseating granulomas showed diffuse staining of the ECM for hyaluronan, intracellular localization of decorin within epithelioid cells, deposition of collagen within the outer rim of granulo-mas and staining for $\alpha$-SMA and type I procollagen (not shown) in a pattern similar to that seen in sarcoidosis.

The localization of versican, mature collagen fibres and sites of synthesis of type I procollagen showed considerable similarities in the granulomas of the three diseases studied. The ECM surrounding the granulomas contained glycosaminoglycans and collagen, and histochemical localization of glycosaminoglycan in all cases corresponded to localization of the proteoglycan versican. In the ECM surrounding granulomas in all three diseases, myofibroblasts in a versican-rich matrix stained positively for type I procollagen. Whereas versican-rich ECM contained little collagen by histochemistry, these areas appeared to be sites of collagen synthesis. Conversely, areas of dense collagen contained little versican. This pattern suggests transient expression of versican, as type I collagen synthesis occurs in the matrix surrounding developing granulomas. There is a spatial and temporal association between versican proteoglycan expression and type I collagen synthesis in all three diseases.

\section{Discussion}

This study demonstrates that the ECM deposited around developing granulomas in sarcoidosis, EAA and TB is glycosaminoglycan-rich, and that these glycosaminoglycans co-localize with the hyaluronan-binding proteoglycan, versican. Hyaluronan co-localizes with versican, suggesting that this matrix may contain hyaluronan and versican as a macromolecular aggregate. These versicanrich areas contain myofibroblasts that stain positively for type I procollagen. Dense collagenous areas contain little versican. We conclude that in granulomatous fibrotic lung diseases, myofibroblasts synthesize collagen within a specialized ECM rich in versican. Our observations suggest that versican is removed from the matrix concomitant with collagen organization rather than being incorporated into collagen fibres. These findings extend our studies in nongranulomatous lung fibrosis, in which we showed a similar spatial and temporal association between versican proteoglycan expression in the matrix and cellular synthesis of type I procollagen [6]. 

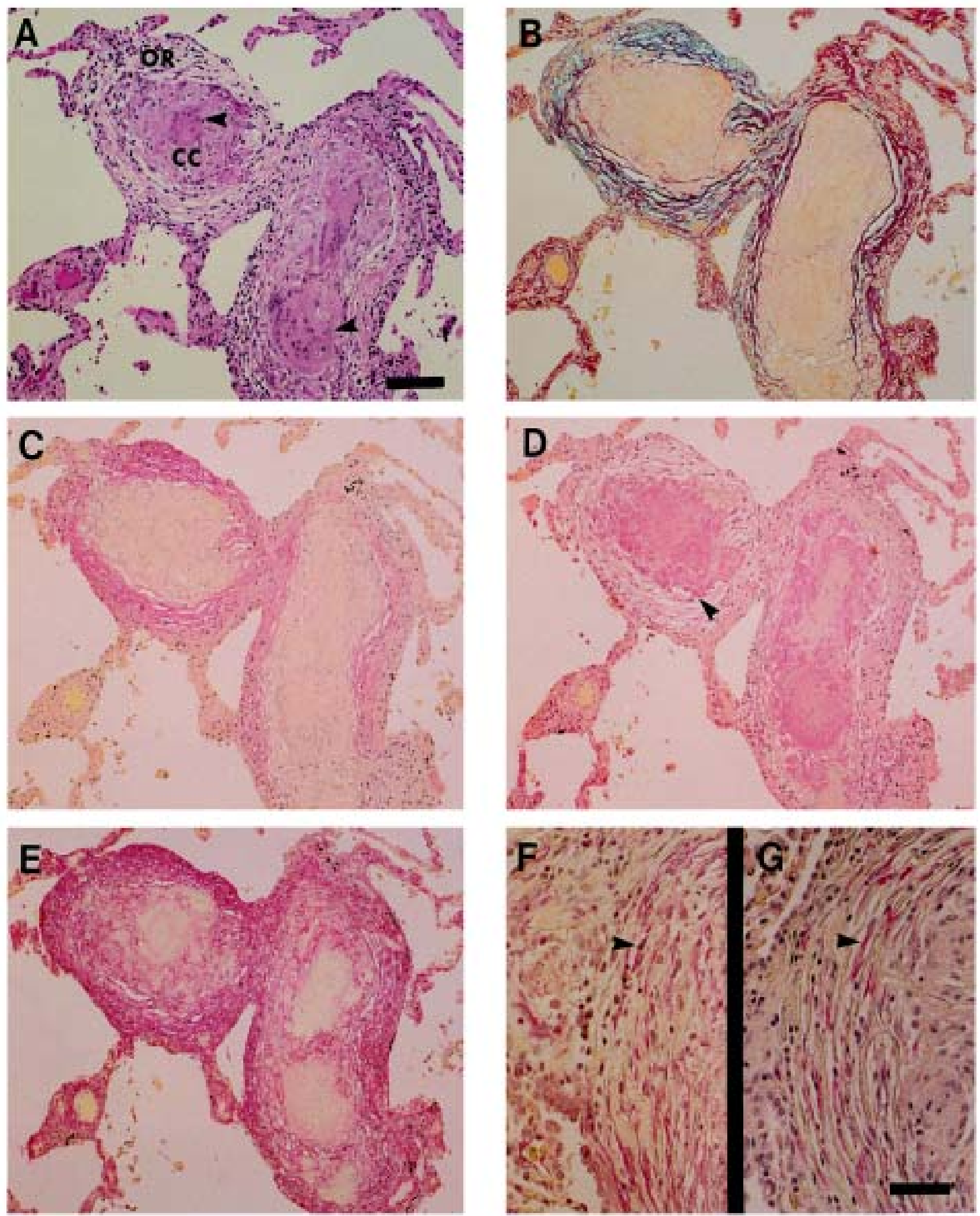

Fig. 1. - Proteoglycan localization in sarcoidosis. A: haematoxylin-eosin stain showing single and coalescing non-necrotizing granulomas composed of a central core (CC) of epithelioid cells, multinucleated giant cells (arrow) and an outer rim (OR) of connective tissue, lymphocytes and myofibroblasts; B: alcian blue-picrosirius red stain showing glycosaminoglycans (blue) localizing to the outer rim of connective tissue while collagen (red) is seen in a concentric pattern within the surrounding rim; C: serial section with (B) showing the co-localization of versican (red) with glycosaminoglycans in the outer rim; D: intracellular staining for decorin is seen within the epithelioid cells (arrow) of the central core of the granulomas; E: extracellular staining for hyaluronan is seen within the extracellular matrix (ECM) of the central core, the ECM of the outer rim and the adjacent interstitium; $\mathrm{H}$ : high power view of the outer rim of connective tissue showing alpha-smooth muscle actin positive (red) myofibroblasts (arrow); G: the corresponding field showing myofibroblasts (arrow) positive for type I procollagen (red). Internal scale bars: panels A-E, $100 \mu \mathrm{m}$; panels F and G, $50 \mu \mathrm{m}$. 

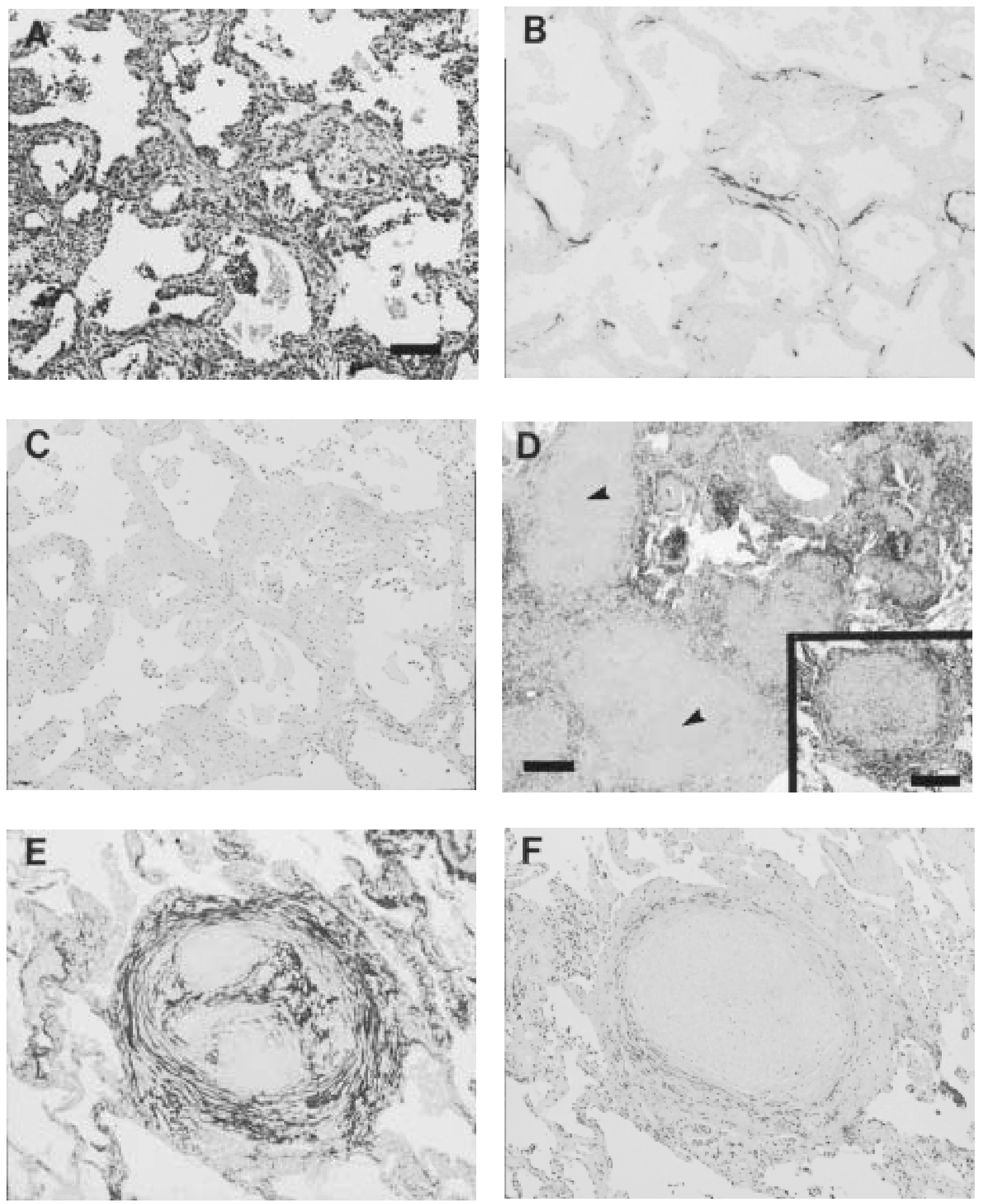

Fig. 2. - Proteoglycan localization in extrinsic allergic alveolitis (A, B, C) and in tuberculosis (D, E, F). A: haematoxylin-eosin stain showing interstitial pneumonitis with no granulomas seen in this field; B: alcian blue-picrosirius red stain showing glycosaminoglycans (blue) localizing to the thickened interstitium with minimal collagen (red) deposition seen within the interstitium; C: same field as in (B) showing the localization of versican (red) in the thickened interstitium; D: haematoxylin-eosin stain of the granulomas lesions from a case of tuberculosis showing caseous necrosis (arrows) and a granuloma without caseation (inset); E: alcian blue-picrosirius red stain of the noncaseating granuloma seen in panel A inset, glycosaminoglycans (blue) are seen in the outer rim of connective tissue and collagen (red) staining is seen in a concentric pattern similar to that of sarcoidosis; F: same field as in E showing the co-localization of versican (red) to the same area as the glycosaminoglycans. Internal scale bars: panels AC, E and F, $100 \mu \mathrm{m}$; panel D $300 \mu \mathrm{m}$, inset panel D $200 \mu \mathrm{m}$. 
Versican is a large chondroitin sulphate-containing proteoglycan produced by smooth muscle cells and fibroblasts [5]. Versican has been shown to be expressed transiently at sites of mesenchymal cell differentiation in skeletal development [11] and in arterial walls, early in atherosclerosis [12], consistent with functions associated with proliferation, migration and matrix synthesis by connective tissue cells. Though the exact function of versican is not known, there is evidence for a number of putative functions for versican. The N-terminal region of the core protein of versican binds hyaluronan [7], and versican's structure is similar to that of the hyaluronan-binding proteoglycan aggrecan, which forms the hydrated matrix of cartilage $[5,13]$. Thus, versican may influence the water content of the ECM at sites of early remodelling, and may facilitate the formation of an open oedematous matrix in which cell migration and proliferation occur. Versican inhibits the adhesion of a number of cell types to the matrix [14, 15], a property that may facilitate cell migration or changes in architecture at sites of remodelling. Additional functions for proteoglycan-bound glycosaminoglycans are likely. The binding and localization of a number of cytokines, growth factors and enzymes is well established for heparan sulphate. It is possible that chondroitin sulphate bound to versican may participate in analogous interactions, though this remains to be studied [5]. Although the exact role of versican in lung repair remains unknown, our results are consistent with a function for versican in the cell biology of myofibroblast proliferation and/or collagen accumulation common to both granulomatous and nongranulomatous lung conditions.

Hyaluronan is distributed throughout the ECM in sarcoidosis, EAA and TB resulting in a diffuse pattern analogous to that seen in the lesions of the nongranulomatous conditions: IPF; ARDS; and BOOP. These findings are consistent with studies demonstrating increased hyaluronan levels in the bronchoalveolar lavage fluid of patients with sarcoidosis [16], EAA [17], ARDS [18], IPF [19] and experimental bleomycin-induced alveolitis [20]. The synthesis and deposition of hyaluronan in experimental bleomycin-induced fibrosis is an early event which preceeds collagen deposition [20] and is related to the early inflammatory response following lung injury [21]. Hyaluronan is thought to play a role in regulation of fluid balance within the interstitium $[22,23]$ which in inflammatory conditions may lead to an open oedematous matrix which facilitates cell migration, Cell migration may be faciltated by the binding of specific cell surface receptors to hyaluronan [24] as occurs in healing wounds [24]. The hyaluronan receptor CD44 is expressed on pulmonary macrophages and epithelial cells [25].

In granulomatous lung conditions we found that the small leucine-rich proteoglycan decorin is found primarily intracellularly within epithelioid cells and some macrophages, myofibroblasts and bronchial epithelial cells. We have previously shown similar intracellular localization of decorin in the nongranulomatous conditions IPF, ARDS and BOOP [6]. Decorin is a small interstitial proteoglycan which is usually found in close association with collagen fibrils $[26,27]$, has been shown to influence collagen fibrillogenesis in vitro [28], and is believed to be directly involved in collagen deposition. Unlike versican, decorin appears to be a component of mature collagen fibres in the human lung. Decorin can bind and inhibit the action of transforming growth factor (TGF)- $\beta$ [29], a cytokine thought to have a key role in fibrotic conditions such as IPF [30]. In sarcoidosis [31] and in post-burn hypertrophic scars [32] decorin has been shown to co- localize with TGF- $\beta$. This association between decorin and TGF- $\beta$ may facilitate clearance or inactivation of TGF- $\beta$, and thereby modulate deposition of ECM. In an animal model of glomerulonephritis driven by TGF- $\beta$, the i.v. administration of decorin reduced the deposition of ECM components [33]. Biglycan is similar to decorin in structure [9], can also bind and inhibit TGF- $\beta$ [29] but does not bind to collagen [27].

This study demonstrates that versican deposition is a consistent feature of granulomatous inflammation produced by different aetiological agents. Versican is associated with myofibroblasts that are actively synthesizing collagen, and appears to be an important component of a provisional matrix in which early collagen synthesis takes place. We have previously reported similar findings in nongranulomatous lung conditions [6] and postulate that versican deposition is a common feature of the repair phase of both granulomatous and nongranulomatous inflammatory processes. The presence of versican in areas where dense collagen deposition and extensive remodelling of the parenchyma have yet to occur, suggests that versican influences the process of matrix remodelling following lung injury. Further studies are needed to delineate the functions of versican in the cell biology of pulmonary fibrosis.

\section{References}

1. Adams DO. The granulomatous inflammatory response. Am J Pathol 1976; 84: 164-191.

2. Hogg JC. Chronic interstitial lung disease of unknown cause: a new classification based on pathogenesis. AJR 1991; 156: 225-233.

3. Peyrol S, Takiya C, Cordier J, Grimaud J. Organization of the connective matrix of the sarcoid granuloma. Ann $N Y$ Acad Sci 1986; 465: 268-285.

4. Bateman E, Turner-Warwick M, Adelmann-Grill BC. Immunohistochemical study of collagen types in human foetal lung and fibrotic lung disease. Thorax 1981; 36: 645-653.

5. Roberts CR, Wight TN, Hascall VC. Proteoglycans. In: Crystal RG, West JB, Weibel E, Barnes PJ, eds. The Lung: Scientific Foundations, second edition. Philadelphia, Lippincott-Raven, 1997; pp. 757-767.

6. Bensadoun ES, Burke AK, Hogg JC, Roberts CR. Proteoglycan deposition in lung fibrosis. Am J Respir Crit Care Med 1996; 154: 1819-1828.

7. LeBaron RG, Zimmermann DR, Ruoslahti E. Hyaluronate binding properties of versican. J Biol Chem 1992; 267: 10003-10010.

8. Fisher LW, Hawkins GR, Tuross N, Termine JD. Purification and partial characterization of small proteoglycans I and II, bone sialoproteins I and II, and osteonectin from the mineral compartment of developing human bone. $J$ Biol Chem 1987; 262: 9702-9708.

9. Fisher LW, Termine JD, Young MF. Deduced protein sequence of bone small proteoglycan I (biglycan) shows homology with proteoglycan II (decorin) and several nonconnective tissue proteins in a variety of species. $J$ Biol Chem 1989; 264: 4571-4576.

10. Adler KB, Low RB, Leslie KO, Mitchell J, Evans JN. Contractile cells in normal and fibrotic lung. Lab Invest 1989; 60: 473-485. 
11. Shinomura T, Jensen K, Yamagata M, Kimata K, Solursh M. The distribution of mesenchyme proteoglycan (PGM) during wing bud outgrowth. Anat Embryol 1990; 181: 227-233.

12. Wight TN. Cell biology of arterial proteoglycans. Arteriosclerosis 1989; 9: 1-20.

13. Naso MF, Zimmermann DR, Iozzo RV. Characterization of the complete genomic structure of the human versican gene and functional analysis of its promoter. J Biol Chem 1994; 269: 32999-33008.

14. Yamagata K, Suzuki S, Akiyama SK, Yamada KM, Kimata K. Regulation of cell-substrate adhesion by proteoglycans immobilized on extracellular substrates. J Biol Chem 1989; 264: 801-806.

15. Yamagata M, Kimata K. Repression of a malignant cellsubstratum adhesion phenotype by inhibiting the production of the anti-adhesive proteoglycan, PG-M/versican. $J$ Cell Sci 1994; 107: 2581-2590.

16. Hallgren R, Eklund A, Engstrom-Laurent A, Schmekel B. Hyaluronate in bronchoalveolar lavage fluid: a new marker in sarcoidosis reflecting pulmonary disease. $B M J$ 1985; 290: 1778-1781.

17. Bjermer L, Engstrom-Laurent A, Lundgren R, Rosenhall L, Hallgren R. Hyaluronate and type III procollagen peptide concentrations in bronchoalveolar lavage fluid as markers of disease activity in farmer's lung. $\mathrm{Br}$ Med $\mathrm{J}$ 1987; 295: 803-806.

18. Bjermer L, Lundgren R, Hallgren R. Hyaluronan and type III procollagen peptide concentrations in bronchoalveolar lavage fluid in idiopathic pulmonary fibrosis. Tho$\operatorname{rax} 1989$; 44: 126-131.

19. Hallgren R, Samuelsson T, Laurent TC, Modig J. Accumulation of hyaluronan (hyaluronic acid) in the lung in adult respiratory distress syndrome. Am Rev Respir Dis 1989; 139: 682-687.

20. Nettelbladt O, Hallgren R. Hyaluronan (hyaluronic acid) in bronchoalveolar lavage fluid during the development of bleomycin-induced alveolitis in the rat. Am Rev Respir Dis 1989; 140: 1028-1032.

21. Anderson Bray B, Sampson PM, Osman M, Giandomenico A, Turino GM. Early changes in lung tissue hyaluronan (hyaluronic acid) and hyaluronidase in bleomycin-induced alveolitis in hamsters. Am Rev Respir Dis 1991; 143: 284288.
22. Nettelbladt O, Tengblad A, Hallgren R. Lung accumulation of hyaluronan parallels pulmonary edema in experimental alveolitis. Am J Physiol 1989; 257: L379-L384.

23. Bhattacharya J, Cruz T, Bhattacharya S, Bray BA. Hyaluronan affects extravascular water in lungs of unanaesthetized rabbits. J Appl Physiol 1989; 66: 2595-2599.

24. Laurent TC, Fraser JRE. Hyaluronan. FASEB J 1992; 6: 2397-2404.

25. Alho AM, Underhill CB. The hyaluronate receptor is preferentially expressed on proliferating epithelial cells. $J$ Cell Biol 1989; 108: 1557-1565.

26. van Kuppevelt TH, Cremers FP, Domen JG, van Beuningen HM, van den Brule AJ, Kuyper CM. Ultrastructural localization and characterization of proteoglycans in human lung alveoli. Eur J Cell Biol 1985; 36: 74-80.

27. Fleischmajer R, Fisher LW, MacDonald ED, Jacobs L, Perlish JS, Termine JD. Decorin interacts with fibrillar collagen of embryonic and adult human skin. J Struct Biol 1991; 106: 82-90.

28. Vogel KG, Paulsson M, Heinegärd D. Specific inhibition of type I and type II collagen fibrillogenesis by the small proteoglycan of tendon. Biochem J 1984; 223: 587-597.

29. Yamaguchi Y, Mann DM, Ruoslahti E. Negative regulation of transforming growth factor-beta by the proteoglycan decorin. Nature 1990; 346: 281-284.

30. Khalil N, O'Connor RN, Unrnh HW, et al. Increased production and immunohistochemical localization of transforming growth factor-beta in idiopathic pulmonary fibrosis. Am J Respir Cell Mol Biol 1991; 5: 155-162.

31. Limper AH, Colby TV, Sanders MS, Asakura S, Roche PC, DeRemee RA. Immunohistochemical localization of transforming growth factor-beta 1 in the nonnecrotizing granulomas of pulmonary sarcoidosis. Am J Respir Crit Care Med 1994; 149: 197-204.

32. Scott PG, Dodd CM, Tredget EE, Ghahary A, Rahemtulla F. Immunohistochemical localization of the proteoglycan decorin, biglycan and versican and transforming growth factor-beta in human post-burn hypertrophic and mature scars. Histopathology 1995; 26: 423-431.

33. Border WA, Noble NA, Yamamoto T, et al. Natural inhibitor of transforming growth factor-beta protects against scarring in experimental kidney disease. Nature 1992; 360: $361-364$. 